\title{
Approximating Fixed Points of Reich-Suzuki Type Nonexpansive Mappings in Hyperbolic Spaces
}

\author{
Kifayat Ullah, ${ }^{1}$ Junaid Ahmad $\mathbb{D}^{1},{ }^{1}$ Manuel De La Sen $\mathbb{D}^{\circ},{ }^{2}$ and Muhammad Naveed Khan ${ }^{1}$ \\ ${ }^{1}$ Department of Mathematics, University of Science and Technology, Bannu 28100, Khyber Pakhtunkhwa, Pakistan \\ ${ }^{2}$ Institute of Research and Development of Processes, University of the Basque Country, Campus of Leioa (Bizkaia), \\ P. O. Box 644-Bilbao, Barrio Sarriena, Leioa 48940, Spain
}

Correspondence should be addressed to Junaid Ahmad; ahmadjunaid436@gmail.com

Received 25 May 2020; Revised 17 June 2020; Accepted 24 June 2020; Published 22 July 2020

Academic Editor: Ali Jaballah

Copyright (c) 2020 Kifayat Ullah et al. This is an open access article distributed under the Creative Commons Attribution License, which permits unrestricted use, distribution, and reproduction in any medium, provided the original work is properly cited.

In this work, we prove some strong and $\Delta$ convergence results for Reich-Suzuki type nonexpansive mappings through $M$ iterative process. A uniformly convex hyperbolic metric space is used as underlying setting for our approach. We also provide an illustrate numerical example. Our results improve and extend some recently announced results of the metric fixed-point theory.

\section{Introduction}

A self-map $S$ on a subset $B$ of a metric space $X=(X, p)$ is called contraction if there exists some constant $\theta \in[0,1)$ such that for all $a, b \in B$ it follows that $p(S a, S b) \leq \theta p(a, b)$. If $p(S a, S b) \leq p(a, b)$ for all $a, b \in B$, then $S$ is called nonexpansive. A point $w \in B$ is called a fixed point of $S$ whenever $w=S w$. Banach [1] theorem (1922) states that any contraction map $S$ on a complete metric space has a unique fixed point which is the limit of the sequence $\left\{x_{k}\right\}$ generated by the Picard iterates, that is, $x_{k+1}=S x_{k}$. In 1965, Kirk [2], Browder [3], and Göhde [4] independently proved that any self nonexpansive mapping $S$ defined on a bounded closed convex subset $B$ of a uniformly convex Banach space always has a fixed point. Now, a natural question which comes in mind is that whether the sequence $\left\{x_{k}\right\}$ of Picard iterates converges to a fixed point of a self nonexpansive mapping. The answer of this question in general is negative. Therefore, there is a need to construct some new procedures to overcome such situations and to obtain a better rate of convergence, for example, Mann [5], Ishikawa [6], Noor [7], S [8], Abbas and Nazir [9], and Thakur et al. [10] iterative processes are often used to approximate fixed points of nonexpansive mappings. In 2008, Suzuki [11] introduced a weaker notion of nonexpansive mappings: a self-map $S$ on a subset $B$ of a metric space is said to be Suzuki type nonexpansive if for every two elements $a, b$ in $B, p(S a, S b) \leq p(a, b)$ holds whenever $(1 / 2) p(a, S a) \leq p(a, b)$. It is easy to observe that the class of Suzuki type nonexpansive mappings properly includes the class of nonexpansive mappings.

The class of Suzuki type nonexpansive mappings was studied extensively by many authors (cf. [10, 12-21]). Very recently, Pant and Pandey [22] introduced Reich-Suzuki type nonexpansive mappings which in turn include the class of Suzuki type nonexpansive mappings.

Definition 1 (see [22]). Let $B$ be a nonempty subset of a metric space. A map $S: B \longrightarrow B$ is said to be Reich-Suzuki type nonexpansive if for all $a, b \in B$, there is some constant $t \in[0,1)$ such that

$$
\begin{aligned}
\frac{1}{2} p(a, S a) & \leq p(a, b) \Longrightarrow p(S a, S b) \\
& \leq t p(a, S a)+t p(b, S b)+(1-2 t) p(a, b) .
\end{aligned}
$$

Approximation of fixed points of nonexpansive and generalized nonexpansive mappings is an active area of research on its own [23-27]. Recently, Pant and Pandey [22] used Thakur et al. [10] iterative process to approximate fixed points of Reich-Suzuki type nonexpansive mappings. The purpose of this paper is to prove strong and $\Delta$ convergence results for Reich-Suzuki type nonexpansive mappings under $M$ iterative process [12], which is known to converge faster 
than the Thakur et al. [10] iterative process. In this way, we improve and extend to many results of the current literature.

Kohlenbach [28] suggested the concept of generalized metric spaces and so-called hyperbolic metric spaces. This type of metric spaces includes normed spaces, the Hilbert ball with the hyperbolic metric, Cartesian products of Hilbert balls, metric trees, Hadamard manifolds, and CAT (0) spaces in the sense of Gromov. The definition is given as follows:

A triplet $(X, p, \mathbb{U})$ is called a hyperbolic metric space whenever $(X, p)$ is a metric space and $\mathbb{H}: X \times X \times[0,1] \longrightarrow X$ is a function such that for all $a, b, w, s \in X$ and $\mu, \xi \in[0,1]$, the following conditions hold.

$$
\begin{aligned}
&\left(C_{1}\right) p(s, \mathbb{H}(a, b, \mu)) \leq(1-\mu) p(s, a)+\mu p(s, b) \\
&\left(C_{2}\right) p(\mathbb{H}(a, b, \mu), \mathbb{H}(a, b, \xi))=|\mu-\xi| p(a, b) \\
&\left(C_{3}\right) \mathbb{H}(a, b, \mu)=\mathbb{H}(b, a, 1-\mu) \\
&\left(C_{4}\right) p(\mathbb{H}(a, s, \mu), \mathbb{H}(b, w, \mu)) \leq \mu p(s, w)+ \\
&(1-\mu) p(a, b)
\end{aligned}
$$

The set $\operatorname{seg}[a, b]:=\{\mathbb{H}(a, b, \mu): \mu \in[0,1]\}$ is known as metric segment with endpoints $a$ and $b$. Throughout, we will write $\mathbb{H}(a, b, \mu)=(1-\mu) a \oplus \mu b$. A subset $B$ of $X$ is called convex provided that $(1-\mu) a \oplus \mu b \in B$, for every $a, b \in B$ and $\mu \in[0,1]$. When there is no ambiguity, we will write $(X, p)$ for $(X, p, \mathbb{H})$.

Definition 2 Let $(X, p)$ be a hyperbolic metric space. For any $s \in X, c>0$ and $\lambda>0$. Set

$$
\begin{gathered}
\sigma(c, \lambda)=\inf \left\{1-\frac{1}{c} p\left(\frac{1}{2} a \oplus \frac{1}{2} b, s\right): p(a, s) \leq c,\right. \\
p(b, s) \leq c, p(a, b) \geq c \lambda\} .
\end{gathered}
$$

We say that $X$ is uniformly convex whenever $\sigma(c, \lambda)>0$, for every $c>0$ and $\lambda>0$.

Definition 3 (see $[29,30])$. A hyperbolic metric space $(X, p)$ is said to be strictly convex provided that for every $a, b, s \in X$ and $\mu \in(0,1)$ such that $p(\mu a \oplus(1-\mu) b, s)=p(a, s)=$ $p(b, s)$ follows the condition $a=b$. By [30], every uniformly convex hyperbolic metric space is strictly convex.

Recently, Ullah and Arshad [12] introduced $M$-iteration process in Banach spaces. The hyperbolic space version of $M$ iteration process reads as follows:

$$
\left.\begin{array}{l}
x_{1}=x \in B, \\
z_{k}=\left(1-\delta_{k}\right) x_{k} \oplus \delta_{k} S x_{k}, \\
y_{k}=S z_{k}, \\
x_{k+1}=S y_{k}, \\
k \geq 1,
\end{array}\right\}
$$

where $\left\{\delta_{k}\right\}_{k=1}^{\infty}$ is a sequence in $(0,1)$. Ullah and Arshad [12] proved some weak and strong convergence results of $M$ iterative process for Suzuki type nonexpansive mappings in the context of Banach spaces. Ullah et al. [31] extended the results of Ullah and Arshad [12] to the setting of CAT (0) spaces. In this paper, we study $M$ iteration process for Reich-Suzuki type nonexpansive mappings in the setting of hyperbolic spaces.

\section{Preliminaries}

Let $B$ be a nonempty subset of hyperbolic metric space $(X, p)$ and $\left\{x_{k}\right\}$ be a bounded sequence in $X$. For each $a \in X$, define

(i) Asymptotic radius of $\left\{x_{k}\right\}$ at $a$ as

$$
r\left(\left\{x_{k}\right\}, a\right):=\limsup _{k \longrightarrow \infty} p\left(x_{k}, a\right) .
$$

(ii) Asymptotic radius of $\left\{x_{k}\right\}$ relative to $B$ as

$$
r\left(\left\{x_{k}\right\}, B\right):=\inf \left\{r\left(\left\{x_{k}\right\}, a\right) ; a \in B\right\} .
$$

(iii) Asymptotic center of $\left\{x_{k}\right\}$ relative to $K$ by

$$
A\left(\left\{x_{k}\right\}, B\right):=\left\{a \in B: r\left(\left\{x_{k}\right\}, a\right)=r\left(\left\{x_{k}\right\}, B\right)\right\} .
$$

We know that in a complete hyperbolic space with monotone modulus of uniform convexity, every bounded sequence $\left\{x_{k}\right\}$ has a unique asymptotic center with respect to every nonempty closed convex subset $B$ of $X$.

Definition 4 (see [32]). Assume that $\left\{x_{k}\right\}$ is a bounded sequence in a hyperbolic space $(X, p)$. Then, $\left\{x_{k}\right\}$ is said to be $\Delta$-convergent to a point $s \in X$, if $s$ is a unique asymptotic center of each subsequence $\left\{x_{k_{i}}\right\}$ of $\left\{x_{k}\right\}$.

The following lemma gives many numbers of ReichSuzuki type nonexpansive mappings.

Lemma 1 (see [22]). Let $B$ be a nonempty subset of a hyperbolic space and $S: B \longrightarrow B$. If $S$ is Suzuki nonexpansive, then $S$ is Reich-Suzuki type nonexpansive with constant $t=0$.

Fixed-point set structure of Reich-Suzuki type maps is as follows.

Lemma 2 (see [22]). Let $B$ be a nonempty subset of a hyperbolic space and $S: B \longrightarrow B$. If $S$ is Reich-Suzuki type nonexpansive, then $F(S)$ is closed. Furthermore, if the space $X$ is strictly convex and the set $B$ is convex, then $F(S)$ is also convex.

Lemma 3 (see [22]). Let $B$ be a nonempty subset of a hyperbolic metric space and $S: B \longrightarrow B$ is a Reich-Suzuki type nonexpansive. Then, for every $a \in B$ and $w \in F(S)$, it implies that $p(S w, S a) \leq p(w, a)$.

Lemma 4 (see [22]). Let $B$ be a nonempty subset of a hyperbolic metric space and $S: B \longrightarrow B$ is a Reich-Suzuki type nonexpansive. Then, for all $a, b \in B$, it follows that

$$
p(a, S b) \leq\left(\frac{3+t}{1-t}\right) p(a, S a)+p(a, b) .
$$

Lemma 5 (see [33]). Let $(X, p)$ be a complete hyperbolic space with a monotone modulus of uniform convexity and $x \in X$. If $0<a \leq \delta_{k} \leq b<1$ and $\left\{x_{k}\right\},\left\{y_{k}\right\}$ are sequences in $X$ such that $\limsup _{k \rightarrow \infty} d\left(x_{k}, x\right) \leq \xi, \limsup _{k \rightarrow \infty} p\left(y_{k}, x\right) \leq \xi$, and $\lim _{k \rightarrow \infty} p\left(\delta_{k} x_{k} \oplus\left(1-\delta_{k}\right) y_{k}, x\right)=\xi$ for some $\xi \geq 0$, then $\lim _{k \longrightarrow \infty} p\left(x_{k}, y_{k}\right)=0$. 


\section{Convergence Results in Hyperbolic Spaces}

Throughout this section, the letter $X$ will stand for a hyperbolic space with a monotone modulus of uniform convexity.

Lemma 6. Let $B$ be a nonempty closed convex subset of $X$. Let $S: B \longrightarrow B$ be a Reich-Suzuki type nonexpansive map with $F(S) \neq \varnothing$. Let $\left\{x_{k}\right\}$ be the sequence generated by (3). Then, $\lim _{k \rightarrow \infty} p\left(x_{k}, w\right)$ exists for $\forall w \in F(S)$.

Proof. Let $w \in F(S)$. By Lemma 3, we have

$$
\begin{aligned}
p\left(x_{k+1}, w\right) & =p\left(S y_{k}, w\right) \leq p\left(y_{k}, w\right) \\
& =p\left(S z_{k}, w\right) \leq p\left(z_{k}, w\right) \\
& =p\left(\left(1-\delta_{k}\right) x_{k} \oplus \delta_{k} S x_{k}, w\right) \\
& \leq\left(1-\delta_{k}\right) p\left(x_{k}, w\right)+\delta_{k} p\left(S x_{k}, w\right) \\
& \leq\left(1-\delta_{k}\right) p\left(x_{k}, w\right)+\delta_{k} p\left(x_{k}, w\right) \\
& =p\left(x_{k}, w\right) .
\end{aligned}
$$

Hence, the sequence $\left\{p\left(x_{k}, w\right)\right\}$ is bounded below and decreasing. Thus, $\lim _{k \longrightarrow \infty} p\left(x_{k}, w\right)$ exists for $\forall w \in F(S)$.

The following theorem will be used in the upcoming results.

Theorem 1. Let $B$ be a nonempty closed convex subset of $X$ and let $S: B \longrightarrow B$ be a Reich-Suzuki type nonexpansive map. Let $\left\{x_{k}\right\}$ be the sequence defined by (3). Then, $F(S) \neq \varnothing$ if and only if $\left\{x_{k}\right\}$ is bounded and $\lim _{k \longrightarrow \infty} p\left(S x_{k}, x_{k}\right)=0$.

Proof. We assume that $\left\{x_{k}\right\}$ is bounded and $\lim _{k \rightarrow \infty} p\left(S x_{k}, x_{k}\right)=0$. Let $w \in A\left(B,\left\{x_{k}\right\}\right)$. We shall prove that $S w=w$. By Lemma 4 , we have

$$
\begin{aligned}
r\left(S w,\left\{x_{k}\right\}\right)= & \limsup _{k \longrightarrow \infty} p\left(x_{k}, S w\right) \\
\leq & \lim \sup _{k \rightarrow \infty}\left(\frac{3+t}{1-t}\right) p\left(S x_{k}, x_{k}\right) \\
& +\lim \sup _{k \rightarrow \infty} p\left(x_{k}, w\right) \\
= & \lim \sup _{k \rightarrow \infty} p\left(x_{k}, w\right)=r\left(w,\left\{x_{k}\right\}\right) .
\end{aligned}
$$

Hence, $r\left(S w,\left\{x_{k}\right\}\right)=r\left(w,\left\{x_{k}\right\}\right)$ and $A\left(B,\left\{x_{k}\right\}\right)$ is singleton set. We must have $S w=w$. Hence, $F(S) \neq \varnothing$.

Conversely, we assume that $F(S) \neq \varnothing$ and $w \in F(S)$. We shall prove that $\left\{x_{k}\right\}$ is bounded and $\lim _{k \rightarrow \infty} p\left(x_{k}, S x_{k}\right)=0$. By Lemma $6, \lim _{k \rightarrow \infty} p\left(x_{k}, w\right)$ exists and $\left\{x_{k}\right\}$ is bounded. Put

$$
\lim _{k \rightarrow \infty} p\left(x_{k}, w\right)=\xi
$$

From the proof of Lemma 6, it follows that

$$
\begin{gathered}
p\left(z_{k}, w\right) \leq p\left(x_{k}, w\right), \\
\Longrightarrow \lim \sup _{k \longrightarrow \infty} p\left(z_{k}, w\right) \\
\leq \lim \sup _{k \longrightarrow \infty} p\left(x_{k}, w\right)=\xi .
\end{gathered}
$$

By Lemma 3, we have

$$
\limsup _{k \rightarrow \infty} p\left(S x_{k}, w\right) \leq \limsup \operatorname{su}_{k \longrightarrow \infty} p\left(x_{k}, w\right)=\xi .
$$

Again from the proof of Lemma 6, it follows that

$$
\begin{aligned}
p\left(x_{k+1}, w\right) & \leq p\left(z_{k}, w\right), \\
\Longrightarrow & \xi \leq \liminf _{k \longrightarrow \infty} p\left(z_{k}, w\right) .
\end{aligned}
$$

From (12) and (15), we get

$$
\xi=\lim _{k \rightarrow \infty} p\left(z_{k}, w\right) .
$$

From (16), we have

$$
\begin{aligned}
& \xi=\lim _{k \longrightarrow \infty} p\left(z_{k}, w\right), \\
& \xi=\lim _{k \longrightarrow \infty} p\left(\left(1-\delta_{k}\right) x_{k} \oplus \delta_{k} S x_{k}, w\right) .
\end{aligned}
$$

Now from (10), (13), and (17) together with Lemma 5, we obtain

$$
\lim _{k \longrightarrow \infty} p\left(S x_{k}, x_{k}\right)=0 .
$$

The $\Delta$ convergence result is as follows.

Theorem 2. Let $B$ be a nonempty closed convex subset of $X$. If $S: B \longrightarrow B$ is a Reich-Suzuki type nonexpansive mapping with $F(S) \neq \varnothing$, then $\left\{x_{k}\right\}$ is defined by (3). $\Delta$ converges to an element of $F(S)$.

Proof. By Theorem 1, the sequence $\left\{x_{k}\right\}$ is bounded. Hence, one can find a $\Delta$-convergent subsequence of $\left\{x_{k}\right\}$. Next, it is our aim to prove that each $\Delta$-convergent subsequence of $\left\{x_{k}\right\}$ has a unique $\Delta$ limit in the set $F(S)$. For this purpose, we assume that $\left\{x_{k}\right\}$ has two $\Delta$-convergent subsequences, namely, $\left\{x_{k_{j}}\right\}$ and $\left\{x_{k_{l}}\right\}$, with $\Delta$ limits $d_{1}$ and $d_{2}$, respectively. In view of Theorem 1 , the sequence $\left\{x_{k_{j}}\right\}$ is bounded and $\lim _{j \rightarrow \infty} p\left(S x_{k_{j}}, x_{k_{j}}\right)=0$. We claim that $d_{1} \in F(S)$. Now,

$$
r\left(\left\{x_{k}\right\}, S d_{1}\right)=\lim \sup _{k \rightarrow \infty} p\left(x_{k}, S d_{1}\right) .
$$

Using Lemma 4, we have

$$
\begin{aligned}
r\left(\left\{x_{k_{j}}\right\}, S d_{1}\right)= & \limsup _{j \rightarrow \infty} p\left(x_{k_{j}}, S d_{1}\right) \\
\leq & \frac{(3+t)}{(1-t)} \limsup _{j \rightarrow \infty} p\left(x_{k_{j}}, S x_{k_{j}}\right) \\
& +\limsup _{j \rightarrow \infty} p\left(x_{k_{j}}, d_{1}\right) \\
\leq & r\left(\left\{x_{k_{j}}\right\}, d_{1}\right) .
\end{aligned}
$$

Since the asymptotic center of $\left\{x_{k_{j}}\right\}$ has a unique element, $S d_{1}=d_{1}$. Similarly, $S d_{2}=d_{2}$. By the uniqueness of asymptotic center of a sequence, we have 


$$
\begin{aligned}
& \lim \sup _{k \rightarrow \infty} p\left(x_{k}, d_{1}\right) \\
& \quad=\limsup _{j \rightarrow \infty} p\left(x_{k_{j}}, d_{1}\right)<\lim \sup _{j \rightarrow \infty} p\left(x_{k_{j}}, d_{2}\right) \\
& =\lim \sup _{k \rightarrow \infty} p\left(x_{k}, d_{2}\right)=\lim \sup _{l \rightarrow \infty} p\left(x_{k_{l}}, d_{2}\right) \\
& \quad<\lim \sup _{l \longrightarrow \infty} p\left(x_{k_{l}}, d_{1}\right)=\lim \sup _{k \rightarrow \infty} d\left(x_{k}, d_{1}\right),
\end{aligned}
$$

which is a contradiction. Hence, the conclusions are reached.

Now, we establish a strong convergence theorem for Reich-Suzuki type nonexpansive maps using iteration process (3).

Theorem 3. Let $B$ be a nonempty compact convex subset of $X$ and $S: B \longrightarrow B$ be a Reich-Suzuki type nonexpansive map with $F(S) \neq \varnothing$. If $\left\{x_{k}\right\}$ is generated by (3), then $\left\{x_{k}\right\}$ converges strongly to the fixed point of $S$.

Proof. By Theorem 1, $\lim _{k \longrightarrow \infty} p\left(S x_{k}, x_{k}\right)=0$. By the compactness assumption, there exists a subsequence $\left\{x_{k_{i}}\right\}$ of $\left\{x_{k}\right\}$ such that $\left\{x_{k_{i}}\right\}$ converges strongly to some $z$ in $B$. By Lemma 4, we have

$$
p\left(x_{k_{i}}, S z\right) \leq\left(\frac{3+t}{1-t}\right) p\left(x_{k_{i}}, S x_{k_{i}}\right)+p\left(x_{k_{i}}, z\right) \longrightarrow 0
$$

By the uniqueness of limits in metric spaces, we must have $z=S z$. By Lemma $6, \lim _{k \longrightarrow \infty} p\left(x_{k}, z\right)$ exists, and hence $z$ is the strong limit of $\left\{x_{k}\right\}$.

Example 1. Let $B=[-2,2]$ be a subset of $\mathbb{R}$ endowed with the usual metric, that is, $p(a, b)=|a-b|$. Define $S: B \longrightarrow B$ by

$$
S a=\left\{\begin{aligned}
-\frac{a}{2}, & \text { if } a \in[-2,0) \backslash\left\{-\frac{1}{4}\right\}, \\
0, & \text { if } a=-\frac{1}{4}, \\
-\frac{a}{4}, & \text { if } a \in[0,2] .
\end{aligned}\right.
$$

Here, first we shall show that the mapping $S$ is not Suzuki type nonexpansive. To do this, we choose $a=-(1 / 4)$ and $b=-(2 / 5)$. The straightforward calculations give

$$
\begin{gathered}
\frac{1}{2} p(a, S a)<p(a, b), \\
p(S a, S b)>p(a, b) .
\end{gathered}
$$

Next, we shall show that the mapping $S$ is Reich-Suzuki type nonexpansive. To do this, we choose $t=1 / 2$. We consider different situations as given below.

Case 1. For $a, b \in[-2,0) \backslash\{-1 / 4\}$, we have

$$
\begin{aligned}
p(S a, S b) & =\frac{1}{2}|a-b| \leq \frac{1}{2}|a|+\frac{1}{2}|b| \leq \frac{3}{4}|a|+\frac{3}{4}|b| \\
& =\frac{1}{2}\left|a+\frac{a}{2}\right|+\frac{1}{2}\left|b+\frac{b}{2}\right| \\
& \leq t p(a, S a)+t p(b, S b)+(1-2 t) p(a, b) .
\end{aligned}
$$

Case 2. For $a, b \in[0,2]$, we have

$$
\begin{aligned}
p(S a, S b) & =\frac{1}{4}|a-b| \leq \frac{1}{4}|a|+\frac{1}{4}|b| \leq \frac{5}{8}|a|+\frac{5}{8}|b| \\
& =\frac{1}{2}\left|a+\frac{a}{4}\right|+\frac{1}{2}\left|b+\frac{b}{4}\right| \\
& =t p(a, S a)+t p(b, S b)+(1-2 t) p(a, b) .
\end{aligned}
$$

Case 3. For $a \in[-2,0) \backslash\{-(1 / 4)\}$ and $b \in[0,2]$, we have

$$
\begin{aligned}
p(S a, S b) & =\left|\frac{a}{2}-\frac{b}{4}\right| \leq \frac{1}{2}|a|+\frac{1}{4}|b| \leq \frac{3}{4}|a|+\frac{5}{8}|b| \\
& =\frac{1}{2}\left|a+\frac{a}{2}\right|+\frac{1}{2}\left|b+\frac{b}{4}\right| \\
& =t p(a, S a)+t p(b, S b)+(1-2 t) p(a, b) .
\end{aligned}
$$

Case 4. For $a \in[-2,0) \backslash\{-(1 / 4)\}$ and $b=(1 / 4)$, we have

$$
\begin{aligned}
p(S a, S b) & =\frac{1}{2}|a| \leq \frac{3}{4}|a| \leq \frac{3}{4}|a|+\frac{1}{8}=\frac{1}{2} p(a, S a)+\frac{1}{2} p(b, S b) \\
& =t p(a, S a)+t p(b, S b)+(1-2 t) p(a, b) .
\end{aligned}
$$

Case 5. For $a \in[-2,0]$ and $b=-(1 / 4)$, we have

$$
\begin{aligned}
p(S a, S b) & =\frac{1}{4}|a| \leq \frac{5}{8}|a| \leq \frac{5}{8}|a|+\frac{1}{8}=\frac{1}{2} p(a, S a)+\frac{1}{2} p(b, S b) \\
& =t p(a, S a)+t p(b, S b)+(1-2 t) p(a, b) .
\end{aligned}
$$

Thus, $S$ is Reich-Suzuki type nonexpansive mapping with $F(S) \neq \varnothing$. Set $\left\{\delta_{k}\right\}=\{1 / k+1\}_{k=1}^{\infty}$. Hence, the requirements of Theorem 2 are fulfilled. Now, the conclusions of Theorems 2 and 3 are reached. However, we cannot directly apply any result in $[9,10,14,16,17,19-22,34]$ and references cited therein because in this situation, $S$ is not Suzuki type nonexpansive mapping.

For the next strong convergence result, compactness assumption is not necessary; however, the following condition will be added.

Definition 5. Let $B$ be a nonempty subset of $X$. A sequence $\left\{x_{k}\right\}$ in $X$ is called Fejer monotone with respect to $B$, if

$$
p\left(x_{k+1}, w\right) \leq p\left(x_{k}, w\right), \quad \text { for every } w \in B \text { and } k \geq 1 \text {. }
$$


A mapping $S: B \longrightarrow B$ is said to satisfy condition $I$ [35] if one can construct a nondecreasing function $\Omega$ : $[0, \infty) \longrightarrow[0, \infty)$ with properties $\Omega(0)=0$ and $\Omega(c)>0$ for every $c \in(0, \infty)$ such that $p(a, S a) \geq \Omega p(a, F(S))$ for each $a \in B$.

The following facts can be found in [34].

Proposition 1. Let $B$ be a nonempty closed subset of $X$. Let $\left\{x_{k}\right\}$ be a Fejer monotone sequence with respect to $B$. Then, $\left\{x_{k}\right\}$ converges strongly to some point of $B$ if and only if $\lim _{k \rightarrow \infty} p\left(x_{k}, B\right)=0$.

Theorem 4. Let $B$ be a nonempty closed convex subset of $X$ and $S: B \longrightarrow B$ be a generalized Reich-Suzuki type nonexpansive map with $F(S) \neq \varnothing$. If $S$ satisfies condition (I), then $\left\{x_{k}\right\}$ generated by (3) converges strongly to the fixed point of S.

Proof. From Theorem 1, we have

$$
\lim _{k \longrightarrow \infty} p\left(S x_{k}, x_{k}\right)=0 .
$$

Since the mapping $S$ satisfies the condition $I$, it follows that

$$
\lim _{k \rightarrow \infty} p\left(x_{k}, F(S)\right) .
$$

By Lemma 2, the set $F(S)$ is closed. It follows from Lemma 6 that $\left\{x_{k}\right\}$ is Fejer monotone sequence with respect to the set $F(S)$. The conclusions are based on Proposition 1 .

\section{Conclusions}

(1) Our results extend the corresponding results of Ullah and Arshad [12] in two ways: (i) from the class of Suzuki type nonexpansive maps to the class of Reich-Suzuki type nonexpansive maps and (ii) from Banach spaces to the general setting of hyperbolic spaces.

(2) Our results extend the corresponding results of Ullah et al. [31] in two ways: (i) from the class of Suzuki type nonexpansive maps to the class of Reich-Suzuki type nonexpansive maps and (ii) from CAT (0) spaces to the general setting of hyperbolic spaces.

(3) Our results also extend and improve the corresponding results proved in $[9,10,14,16,17$, 19-22, 34] and references cited therein.

\section{Data Availability}

No data were used to support this study.

\section{Conflicts of Interest}

The authors declare that they have no conflicts of interest.

\section{Authors' Contributions}

All authors contributed equally and significantly in writing this article. All authors read and approved the final manuscript.

\section{Acknowledgments}

The authors are grateful to the Spanish Government for Grant RTI2018-094336-B-I00 (MCIU/AEI/FEDER, UE) and to the Basque Government for Grant IT1207-19.

\section{References}

[1] S. Banach, "Sur les opérations dans les ensembles abstraits et leur application aux équations intégrales," Fundamenta Mathematicae, vol. 3, pp. 133-181, 1922.

[2] W. A. Kirk, "A fixed point theorem for mappings which do not increase distances," The American Mathematical Monthly, vol. 72, no. 9, pp. 1004-1006, 1965.

[3] F. E. Browder, "Nonexpansive nonlinear operators in a Banach space," Proceedings of the National Academy of Sciences, vol. 54, no. 4, pp. 1041-1044, 1965.

[4] D. Göhde, "Zum prinzip der kontraktiven abbildung," Mathematische Nachrichten, vol. 30, no. 3-4, pp. 251-258, 1965.

[5] W. R. Mann, "Mean value methods in iteration," Proceedings of the American Mathematical Society, vol. 4, no. 3, pp. 506-510, 1953.

[6] S. Ishikawa, "Fixed points by a new iteration method," Proceedings of the American Mathematical Society, vol. 44, no. 1, p. 147, 1974.

[7] M. A. Noor, "New approximation schemes for general variational inequalities," Journal of Mathematical Analysis and Applications, vol. 251, no. 1, pp. 217-229, 2000.

[8] R. P. Agarwal, D. O'Regon, and D. R. Sahu, "Iterative construction of fixed points of nearly asymtotically nonexpansive mappings," Journal of Nonlinear and Convex Analysis, vol. 8, pp. 61-79, 2007.

[9] M. Abbas and T. Nazir, "A new faster iteration process applied to constrained minimization and feasibility problems," Matematicki Vesnik, vol. 66, no. 2, pp. 223-234, 2014.

[10] B. S. Thakur, D. Thakur, and M. Postolache, "A new iterative scheme for numerical reckoning fixed points of Suzuki's generalized nonexpansive mappings," Applied Mathematics and Computation, vol. 275, pp. 147-155, 2016.

[11] T. Suzuki, "Fixed point theorems and convergence theorems for some generalized nonexpansive mappings," Journal of Mathematical Analysis and Applications, vol. 340, no. 2, pp. 1088-1095, 2008.

[12] K. Ullah and M. Arshad, "Numerical reckoning fixed points for Suzuki's generalized nonexpansive mappings via new iteration process," Filomat, vol. 32, no. 1, pp. 187-196, 2018.

[13] S. Dhomphongsa, W. Inthakon, and A. Kaewkhao, "Edelstein's method and fixed point theorems for some generalized nonexpansive mappings," Journal of Mathematical Analysis and Applications, vol. 350, no. 1, pp. 12-17, 2009.

[14] W. Phuengrattana, "Approximating fixed points of Suzukigeneralized nonexpansive mappings," Nonlinear Analysis: Hybrid Systems, vol. 5, no. 3, pp. 583-590, 2011.

[15] A. Abkar and M. Eslamian, "A fixed point theorem for generalized nonexpansive multivalued mappings," Fixed Point Theory, vol. 12, no. 2, pp. 241-246, 2011.

[16] M. Basarir and A. Sahin, "On the strong and $\Delta$-convergence of $S$-iteration process for generalized nonexpansive mappings on CAT (0) spaces," Thai Journal of Mathematics, vol. 12, no. 3, pp. 549-559, 2014

[17] B. Nanjaras, B. Panyanak, and W. Phuengrattana, "Fixed point theorems and convergence theorems for Suzuki-generalized 
nonexpansive mappings in CAT (0) spaces," Nonlinear Analysis: Hybrid Systems, vol. 4, no. 1, pp. 25-31, 2010.

[18] K. Ullah and M. Arshad, "New iteration process and numerical reckoning fixed point in Banach spaces," U.P.B. Scientific Bulletin (Series A)., vol. 79, no. 4, pp. 113-122, 2017.

[19] I. Uddin and M. Imdad, "On certain convergence of S-iteration scheme in CAT (0) spaces," Kuwait Journal of Science, vol. 42, no. 2, pp. 93-106, 2015.

[20] I. Uddin and M. Imdad, "Convergence of SP-iteration for generalized nonexpansive mapping in Hadamard spaces," Hacettepe Journal of Mathematics and Statistics, vol. 48, no. 2, pp. 1595-1604, 2018.

[21] I. Uddin and M. Imdad, "Some convergence theorems for a hybrid pair of generalized nonexpansive mappings in CAT (0) spaces," Journal of Nonlinear and Convex Analysis, vol. 16, no. 3, pp. 447-457, 2015.

[22] R. Pant and R. Pandey, "Existence and convergence results for a class of nonexpansive type mappings in hyperbolic spaces," Applied General Topology, vol. 20, no. 1, pp. 281-295, 2019.

[23] T. Abdeljawad, K. Ullah, and J. Ahmad, "Iterative algorithm for mappings satisfying $\left(\mathrm{B}_{\gamma, \mu}\right)$ condition," Journal of Function Spaces, vol. 2020, Article ID 3492549, 7 pages, 2020.

[24] T. Abdeljawad, K. Ullah, J. Ahmad, and N. Mlaiki, "Iterative approximation of endpoints for multivalued mappings in Banach spaces," Journal of Function Spaces, vol. 2020, Article ID 2179059, 5 pages, 2020.

[25] G. A. Okeke, "Iterative approximation of fixed points of contraction mappings in complex valued Banach spaces," Arab Journal of Mathematical Sciences, vol. 25, no. 1, pp. 83-105, 2018.

[26] K. Ullah, F. Ayaz, and J. Ahmad, "Some convergence results of $\mathrm{M}$ iterative process in Banach spaces," Asian-European Journal of Mathematics, vol. 2019, Article ID 2150017, 2019.

[27] K. Ullah, M. S. U. Khan, N. Muhammad, and J. Ahmad, "Approximation of endpoints for multivalued nonexpansive mappings in geodesic spaces," Asian-European Journal of Mathematics, vol. 2019, Article ID 2050141, 2019.

[28] U. Kohlenbach, "Some logical metatheorems with applications in functional analysis," Transactions of the American Mathematical Society, vol. 357, no. 1, pp. 89-129, 2005.

[29] W. Takahashi, "A convexity in metric space and nonexpansive mappings. I," Kodai Mathematical Seminar Reports, vol. 22, no. 2, pp. 142-149, 1970.

[30] H. Fukhar-ud-din and M. A. Khamsi, "Approximating common fixed points in hyperbolic spaces," Fixed Point Theory and Applications, vol. 2014, Article ID 113, 2014.

[31] K. Ullah, H. N. Khan, and M. Arshad, "Numerical reckoning fixed points in CAT (0) spaces," Sahand Communications in Mathematical Analysis (SCMA), vol. 12, no. 1, pp. 97-111, 2018.

[32] W. A. Kirk and B. Panyanak, "A concept of convergence in geodesic spaces," Nonlinear Analysis: Theory, Methods \& Applications, vol. 68, no. 12, pp. 3689-3696, 2008.

[33] A. R. Khan, H. Fukhar-ud-din, and M. A. Ahmad Khan, "An implicit algorithm for two finite families of nonexpansive maps in hyperbolic spaces," Fixed Point Theory and Applications, vol. 2012, no. 1, p. 54, 2012.

[34] P. Chuadchawna, A. Farajzadeh, and A. Kaewcharoen, "Convergence theorems and approximating endpoints for multivalued Suzuki mappings in hyperbolic spaces," Journal of Computational Analysis and Applications, vol. 28, pp. 903-916, 2020.

[35] H. F. Senter and W. G. Dotson, "Approximating fixed points of nonexpansive mappings," Proceedings of the American Mathematical Society, vol. 44, no. 2, p. 375, 1974. 